\title{
EMPATIA: PERFIL DA PRODUÇÃO CIENTÍFICA E MEDIDAS MAIS UTILIZADAS EM PESQUISA
}

\section{Sonia Maria Lourenço de Azevedo}

Universidade Salgado de Oliveira

\author{
Márcia Maria Peruzzi Elia da Mota \\ Universidade Salgado de Oliveira e Universidade do Estado do Rio de Janeiro \\ Marsyl Bulkool Mettrau \\ Universidade Salgado de Oliveira e Universidade Federal Fluminense
}

\begin{abstract}
Resumo
O presente artigo faz uma revisão sistemática da literatura sobre o construto empatia com o objetivo de analisar as publicações neste tema em relação: (a) quantitativo de publicações por ano; (b) áreas das publicações; (c) tipo de estudo empírico; (d) instrumentos mais utilizados nas pesquisas empíricas. Realiza-se esta revisão na literatura nacional utilizando um Programa de busca nos sites acadêmicos no período de 2007 a 2017. A justificativa pela literatura nacional foi para identificar os estudos sobre o construto empatia, as áreas e, os instrumentos mais utilizados em nosso contexto, sendo estes brasileiros ou adaptados para nossa cultura. Os resultados apontaram que o tema "empatia" tem sido investigado em diversos campos e áreas de estudos tais como: psicologia, medicina, enfermagem, odontologia, fisioterapia, fonoaudiologia, educação, engenharia de produção. A maior frequência dos estudos encontra-se na área da psicologia, com uma forte tendência a validação de escala para nossa cultura e na área da saúde.

Palavras-chave: empatia; medida, psicologia; revisão sistemática.
\end{abstract}

\section{EMPATHY: THE PROFILE OF SCIENTIFIC PRODUCTION AND MEASURE MORE USED IN RESEARCH}

\begin{abstract}
The article presents a systematic review of the construct empathy, with the objective of analyzing the publications in this topic in relation to: (a) number of publications per year; (b) areas of publications; (c) kind of study empirical; (d) most used instruments at empirical research. We made a research in the national literature using a search Program in academic sites at the period of 2007 to 2017. We chose the national literature to identify the studies about empathy, the areas, and the instruments most frequently used in our context, being them Brazilian or adapted to our culture. The results appointed that the topic "empathy" have been investigated in several fields and areas of study, such as psychology, medicine, nursing, dentistry, physiotherapy, speech therapy, education, production engineering. We found the biggest frequency of studies at psychology area, with a strong validation trend of scale to our culture and health area.
\end{abstract}

Keywords: empathy; measure; psychology; systematic review. 


\title{
EMPATÍA: PERFIL DE LA PRODUCCIÓN CIENTÍFICA Y MEDIDAS MÁS UTILIZADAS EN INVESTIGACIÓN
}

\begin{abstract}
Resumen
El presente artículo hace una revisión sistemática de la literatura sobre el constructo empatía con el objetivo de analizar las publicaciones en este tema en relación a: (a) cuantitativo de publicaciones por año; (b) áreas de publicaciones; (c) tipo de estudio, empírico; (d) instrumentos más utilizados en las investigaciones empíricas. Se realiza esta revisión en la literatura nacional utilizando un Programa de búsqueda en los sitios académicos en el período de 2007 a 2017. La justificación por la literatura nacional fue identificar los estudios sobre el constructo empatía, las áreas y los instrumentos más utilizados en nuestro contexto, siendo estos brasileños o adaptados para nuestra cultura. Los resultados apuntaron que el tema "empatía" ha sido investigado en diversos campos y áreas de estudios tales como: psicología, medicina, enfermería, odontología, fisioterapía, fonoaudiología, educación, ingeniería de producción. La mayor frecuencia de los estudios se encuentra en el área de la psicología, con una fuerte tendencia a validación de escala para nuestra cultura, y en el área de la salud.

Palabras clave: empatía; medida, psicología; revisión sistemática.
\end{abstract}

\section{INTRODUÇÃO}

A empatia é um construto psicológico importante para a vida das pessoas e sua influência social vem sendo estudada por diversos autores e pesquisadores da psicologia e ciências sociais (Davis \& Oathout, 1992; Falcone, 1999; Goleman, 1995; Sampaio, Camino, \& Roazzi, 2009). Dentre os diversos autores aqui tratados ao longo deste trabalho, destacam-se Goleman (1995), que descreve a empatia como uma habilidade que afeta o ajustamento social do indivíduo, além de Ickes (1997) e Falcone (1999), os quais evidenciam a importância deste construto para a vida pessoal e profissional de indivíduos.

Goleman (1995) destaca que a empatia é uma habilidade importante que afeta o ajustamento social das pessoas, em todas as fases de sua vida, como na relação com os pais, com os colegas da escola, os alunos em sala de aula, parceiros e amigos, na relação de namoro (Davis \& Oathout, 1992) e casamento (Ickes \& Simpson, 1997), assim como entre membros de uma equipe de trabalho e demais membros da comunidade a que o indivíduo pertence. Ainda, segundo Goleman (1995), essa capacidade pode ser o aspecto de excelência do que é comumente denominado de inteligência social.

Os diversos estudos citados propõem que as pessoas empáticas tornam as relações mais estáveis e agradáveis, reduzindo o conflito e o rompimento (Davis, 1983a, 1983b; Falcone, 1999; Sampaio, Camino, Roazzi, 2009). De outra forma, pessoas não empáticas demonstram dificuldades relacionadas à inteligência social e podem ser prejudicados nos diversos setores da vida social: trabalho, escola, vida conjugal, amizades, família, além de estarem mais propensos a viver à margem da sociedade.

Apesar das divergências conceituais e metodológicas sobre a empatia, "existe consenso entre os teóricos a respeito da forte influência que a empatia pode exercer nos processos de tomada de decisão, especialmente quando esta se refere às questões ligadas ao cuidado, respeito e moralidade" (Sampaio et al., 
2009, p. 224). Damásio (2009) descreve, ao falar de homeostase social, que "a nossa vida deve ser regulada não só pelos nossos desejos e sentimentos, mas também pela nossa preocupação com os desejos e sentimentos dos outros" ( $p$. 179). Na homeostase social, apontada por Damásio, observa-se a importância do outro, do sentir do outro, da forte influência da empatia nas tomadas de decisões e equilíbrio homeostático no ser humano.

A empatia, e sua definição, vem sendo estudada e construída ao longo da história da Psicologia. Em uma revisão feita por Sampaio et al. (2009) fazem uma retrospectiva de como a empatia foi estudada na psicologia nos aspectos conceituais, teóricos e metodológicos. Os autores apontaram que o interesse pelo tema da empatia remonta ao século XIX. Nessa época a empatia era considerada uma característica do ser humano definida como sendo a capacidade de uma pessoa identificar o que estava na consciência de outra pessoa.

Do início do século XX até a década de 1940, o conceito de empatia foi objeto de reflexões teóricas, inclusive por autores como Freud (1905/2006), mas de poucas investigações empíricas. Ainda no século $X X$, a partir da década de 1950 a empatia passa a ser objeto de estudos empíricos. Ressalta-se a importância de Rogers $(1992,2001)$ que abordou a relação empática na prática terapêutica. Para esse autor, a empatia consistia em se ter consideração empática, conseguir sentir o que o outro sente e aceitar incondicionalmente o outro. A Abordagem Centrada na Pessoa, visão psicoterápica de Rogers, tinha como técnica principal a consideração empática do terapeuta pelo seu cliente. Rogers apontou que a empatia poderia ser desenvolvida, aprendida e assim dedicou o seu estudo.

Ainda na segunda metade do século XX, a Psicologia Social e a Psicologia do Desenvolvimento incluíram a empatia como parte de seus temas de interesse. Na psicologia social a empatia foi estudada vinculada a comportamentos prósociais e na psicologia do desenvolvimento foi estudada sob o enfoque da tomada de perspectiva. Isto é, como as crianças veem como os outros sentem e também como elas reconhecem as emoções do outro (Falcone, 1999; Sampaio et al., 2009).

No final do século XX, Hoffman (1981) definiu a empatia como uma resposta afetiva vicária a outra pessoa, ou seja, ela se exerce através do outro, relaciona-se à situação do outro e não na sua própria situação. Sendo ainda esta resposta humana, universal e corroborada fisiologicamente. Para Hoffmann (1981, 1990) são dois os tipos de ativação empática: (1) a imitação de outras pessoas com movimentos de postura e de expressão facial que, quando realizados, produzem na pessoa, por meio de indicadores internos, uma reação que contribui para compreender e sentir a emoção na própria pessoa; (2) é evidenciado por indicadores de dor ou prazer do outro, que experimentam associações com sensações já vivenciadas pelo observador que resulta em uma reação afetiva empática. Neste sentido, propõe que ajudar evoca uma resposta 
empática de aflição e esta resposta favorece o comportamento de ajuda de modo involuntário e praticamente automático.

Batson, Fultz e Schoenrade (1987) consideram que a empatia evoca duas respostas distintas: empatia e angústia pessoal. A empatia é um comportamento pró-social que leva a pessoa a ajudar o outro. A angústia pessoal é um sentimento negativo, gerada pelo fato de se sentir como o outro. A angústia pessoal leva o sujeito a agir no sentido de ajudar a outra pessoa para aplacar sua própria angústia. Esse é um sentimento egoísta, mas que leva a comportamentos pró-sociais. Assim, a empatia começa a ser vista como um conceito multidimensional, uma vez que diferentes emoções são vivenciadas quando se diz que alguém tem empatia.

A avaliação da empatia é feita por diversos métodos, por exemplo, respostas fisiológicas e análises das expressões faciais (Cox et al., 2012; De Greck et al., 2012). Na utilização de diferentes escalas e questionários, temos os estudos de Davis (1980, 1983a, 1983b), que criou o Interpersonal Reactivity Index (IRI). Partindo do pressuposto de que a empatia é um traço constitucional do sujeito, Davis propôs essa escala com quatro dimensões: consideração empática, angústia pessoal (que fariam parte da componente afetiva) e tomada de consciência e fantasia (que estariam associadas ao componente cognitivo da empatia).

No Brasil, Falcone (1999) ampliou as dimensões utilizadas nas escalas de empatia para três componentes: (a) componente cognitivo - relacionado à capacidade de perceber e compreender, com sensibilidade e acurácia, os sentimentos e as perspectivas emocionais do outro; (b) componente afetivo relacionado ao despertar de sentimentos de compaixão, simpatia e/ou preocupação com o bem estar pelo outro; (c) componente comportamental relacionado à demonstração de que o sentimento do outro foi percebido e entendido, de maneira que o outro se sinta compreendido por ele.

Falcone et al. (2008; 2013) criaram e validaram o Inventário de Empatia (IE) brasileiro. Este instrumento de autorrelato avalia quatro dimensões da empatia: Tomada de Perspectiva (TP), que pode ser definida como capacidade de se entender a perspectiva e os sentimentos do outro; Flexibilidade Interpessoal (FI), que envolve a capacidade para tolerar comportamentos, atitudes e pensamentos muito diferentes ou que provocam frustração; Altruísmo ( $\mathrm{Al}$ ), definido como a capacidade para sacrificar temporariamente os próprios interesses para beneficiar ou ajudar alguém; e Sensibilidade Afetiva (SA), que se refere a sentimentos de compaixão, interesse ou consideração pelo outro. As duas primeiras dimensões do IE representam dimensões cognitivas da empatia e as duas últimas, dimensões afetivas.

As escalas de Davis (1983a, 1983b) e Falcone et al. (2008) não foram as únicas construídas a fim de se pesquisar quanti-qualitativamente a influência do construto empatia na vida pessoal e social dos indivíduos. Foram construídas 
diferentes escalas e questionários que visam atender à análise dos aspectos relacionados a este construto nas diversas áreas de pesquisa e de prática tanto na psicologia, na área da saúde, como na medicina, enfermagem, odontologia fisioterapia, na educação, entre outros.

$\mathrm{Na}$ área da saúde, sobre a importância da relação empática nos atendimentos, destaca-se a importância do estudo de Costa e Azevedo (2010) que realizaram uma pesquisa qualitativa, utilizando entrevista semiestruturada com 323 participantes médicos docentes de uma faculdade de medicina. Os autores consideram que a empatia pode enriquecer a prática médica e cogitam a possibilidade de ensinar a ser empático.

Aponta-se a importância de um estudo recente realizado por Aguiar, Formiga e Cantinilo (2017) que investiga a correlação entre a empatia e a personalidade em estudantes do curso de medicina. Este estudo mostrou que empatia e personalidade se correlacionam e concluiu que determinados fatores da empatia são capazes de predizer fatores da personalidade específicos.

Ressalta-se, ainda, o estudo de Falcone, Gil e Ferreira (2007) que trata da relação da verbalização empática em diferentes abordagens terapêuticas. 0 objetivo desse estudo foi avaliar se o tipo de abordagem adotado pelo terapeuta interfere no conteúdo e na frequência de suas verbalizações empáticas. O estudo apontou a importância da verbalização empática estabelecida entre terapeuta e paciente durante a terapia.

Neste trabalho, realiza-se um levantamento das publicações brasileiras com objetivo de identificar o perfil da produção científica do construto empatia em relação ao tipo de pesquisa, ano de maior publicação científica, instrumentos mais utilizados nas publicações e participantes da pesquisa. Foi realizado um corte temporal dos 10 (dez) últimos anos, incluindo o ano de 2017, para a seleção dos mesmos (2007-2017). O foco pela literatura nacional justifica-se diante do objetivo aqui proposto que foi a busca de estudos sobre o construto empatia em nossa cultura.

\section{MÉTODO}

Utilizou-se o programa Harzing's Publishor Perish que realiza busca de dados, recupera, calcula uma série de métricas de citações por meio de dados brutos encontrados do Google Scholar. Ao realizar uma pesquisa nesse programa, obtém-se uma lista das referências pertinentes à expressão de busca, neste caso o descritor "empatia", onde foi gerada uma estatística de produtividade e de impacto de autores e periódicos (Harzing, 2007). Não se empregou o descritor em inglês empathy, porque o foco principal do artigo foi pesquisar o tema destacado na literatura nacional. 


\section{Procedimento}

A consulta resultou em 998 publicações capturadas, dentro de 22.903 cites do Google Scholar Query quando utilizou-se o descritor "empatia", na busca de dados realizada no período de 2007 a 2017. Cabe esclarecer que 1000 é o número máximo de estudos que o Programa recupera. Ressalta-se que a busca foi realizada em agosto de 2017.

Realizou-se uma planilha com todos os estudos capturados, tendo como objetivo fazer uma seleção das publicações a serem consideradas neste artigo. Para esta seleção foram elencados critérios de exclusão das publicações, a saber: (1) artigos de outros países; (2) citações de livros; (3) artigos que utilizavam o termo empatia sem um estudo relacionado, tratando apenas de comentários sobre a importância de ter empatia, entre outros; (3) reflexões sobre o termo empatia sem estudos empíricos; (4) páginas de Web, sem fundamentação científica comprovada (p.ex., revistas de divulgação, artigos em blogs). Na segunda parte do estudo, foi realizada a leitura dos resumos a fim de excluir os artigos, conforme os critérios de exclusão. Assim, a amostra final do estudo foi composta de 53 artigos e publicações, a partir dos quais se realizou a leitura dos resumos e textos completos com objetivo de analisar as publicações em relação as seguintes categorizações: (a) quantitativo de publicações por ano; (b) áreas de estudo das publicações; (c) instrumentos mais utilizados nas pesquisas empíricas.

\section{RESULTADOS}

Quantitativo de estudos

Após a seleção dos artigos, fez-se a tabulação dos dados com objetivo de verificar o ano de publicação dos artigos. A distribuição de artigos por ano encontra-se na Tabela 1 . De um modo geral, os artigos apresentam-se distribuídos de forma relativamente equivalente nos três primeiros anos pesquisados (2007 a 2009), com um aumento na produção no ano de 2010 . Observa-se ainda que, a partir de 2013, há uma queda na produção dos artigos neste tema.

Tabela 1. Quantitativo de Estudos sobre Empatia por ano

\begin{tabular}{ccr}
\hline Ano & Quantitativo & $\%$ \\
\hline 2007 & 5 & 9,61 \\
2008 & 6 & 11,54 \\
2009 & 7 & 13,46 \\
2010 & 18 & 34,61 \\
2011 & 6 & 11,32 \\
2012 & 5 & 9,61
\end{tabular}




\begin{tabular}{rrr}
2013 & 2 & 3,85 \\
2014 & 0 & - \\
2015 & 1 & 1,92 \\
2016 & 1 & 1,92 \\
2017 & 1 & 1,92 \\
\hline
\end{tabular}

Áreas de estudo das publicações

Quanto à área de estudo (Tabela 2), os resultados apontaram que o tema "empatia" tem sido investigado em diversos campos de estudo, tais como: psicologia, saúde, educação, psicobiologia, interface de ação social e educação, engenharia de produção. Observa-se que a área que apresenta maior quantitativo de estudos publicados é a psicologia e a segunda maior área de publicação foi a saúde.

Tabela 2. Quantitativo de estudos sobre empatia por área

\begin{tabular}{ccr}
\hline Área & Quantitativo & $\%$ \\
\hline Psicologia & 30 & 57,69 \\
Saúde & 17 & 32,69 \\
Educação & 2 & 3,84 \\
Ação Social e Saúde & 1 & 1,92 \\
Engenharia de Produção & 1 & 1,92 \\
Psicobiologia & 1 & 1,92 \\
\hline
\end{tabular}

Nos diversos campos da psicologia, evidenciam-se 30 pesquisas, tais como: psicologia social, do desenvolvimento, da aprendizagem. Ressaltam-se ainda os estudos psicométricos com objetivo de realizar validações e adaptações de escalas para medir o constructo empatia, destacando-se a adaptação da Escala de Davis (1983a, 1983b) com um maior quantitativo de estudos publicados.

$\mathrm{Na}$ área da saúde foram computados 17 artigos realizados nos seguintes campos de pesquisa e atuação: enfermagem, fisioterapia, medicina, odontologia, fonoaudiologia. Consideraram-se, ainda, na categorização saúde: os trabalhos realizados com objetivo de analisar os atendimentos dos hospitais; programas de atendimento, como o Programa de Saúde da Família (PSF), entre outros.

Os dois estudos na área da educação foram realizados com o objetivo de avaliar a qualidade de serviço prestados aos clientes, no caso alunos, de instituições de ensino superior. Oliveira e Ferreira (2008) realizaram a adaptação da Escala Servqual com o objetivo de medir o gap entre as expectativas e as percepções quanto a qualidade do serviço prestados aos estudantes do curso de Engenharia de Produção. Prass, Sant'Anna e Godoy (2010) também utilizaram o modelo da Escala Servqual com o mesmo objetivo em alunos dos cursos de 
Administração, Enfermagem e Psicologia. Ressalta-se que a empatia é um dos 5 determinantes da qualidade de serviço.

$\mathrm{Na}$ categorização ação social e saúde, temos o artigo de Castro, Silva, Monteiro, Palma e Resende (2010) que realiza um estudo sobre os motivos de permanência dos praticantes nos programas de exercícios físicos oferecidos pelo Serviço Social do Comércio. Castro et al. (2010) ressaltam os benefícios da prática regular e adequada de exercício físicos na prevenção de doenças e apontam ainda que o bem-estar pessoal foi considerado o principal motivo alegado pelos participantes dos programas. Ressalta-se que a empatia do professor na relação com os alunos também é um dos motivos alegado da permanência da prática de exercícios do usuário do programa.

Hora, Moura e Vieira (2009) realizam um estudo, na área de engenharia de produção, com objetivo de mensurar a qualidade de serviços internos de um shopping center. Para isso, o estudo utilizou a análise performance importância (API), que trata-se de um questionário de perguntas fechadas, onde os respondentes da pesquisa avaliam a performance em cada um dos itens e, também, a importância que atribuíam a cada critério. O questionário é composto por 20 itens, com 4 dimensões específicas em cada grupo de perguntas, que são: confiabilidade, presteza, garantia, empatia e aspectos tangíveis. Assim, a empatia foi uma das dimensões analisadas.

$\mathrm{Na}$ área de psicobiologia, a dissertação de mestrado de Silva (2016) utilizou um protocolo que propôs avaliar empatia e comportamento pró-social em ratos Wistar. Nesse estudo, o comportamento pró-social refere-se a ações com a intenção de beneficiar o outro, independente do auto benefício. Silva (2016) aponta que "por trás de um comportamento pró-social pode estar um componente emocional motivador que, em seu estudo, exemplificou como sendo a empatia um desses componente" (p. 8).

\section{Instrumentos mais utilizados nas pesquisas empíricas}

$\mathrm{Na}$ Tabela 3 temos o quantitativo de instrumentos utilizados nas pesquisas empíricas com objetivo de investigar o construto empatia. A Escala Servqual, com maior número de estudo, foi desenvolvida por Parasuraman, Zeithami e Berry (1988). Trata-se de um instrumento que objetiva mensurar como os clientes percebem a qualidade de um serviço tendo como base a percepção e a expectativa dos clientes sobre como o serviço prestado está sendo executado, sendo a empatia um dos 5 determinantes da qualidade do serviço.

Observa-se, na área de psicologia, que o instrumento mais utilizado foi o Interpersonal Reactivity Index (IRI) de Davis (1980, 1983a, 1983b), sendo computadas na Tabela 3 as traduções deste instrumento no Brasil que foi denominada de Escala Multidimensional de Reatividade Interpessoal (EMRI), que é uma adaptação do IRI de Davis para a população brasileira com diversos estudos de validação e consistência estrutural (2012, 29 de setembro; Formiga 
et al., 2011; Koller, Camino, \& Ribeiro, 2001; Sampaio, Guimarães, Camino, Formiga, \& Menezes, 2011).

Tabela 3. Quantitativo de instrumentos utilizados para investigar empatia

\begin{tabular}{ccc}
\hline Instrumentos & Quantitativo & $\%$ \\
\hline Escala Servqual & 9 & 23,68 \\
IRI /EMRI & 8 & 21,05 \\
SSRS-BR & 4 & 10,53 \\
Escala de Empatia de Bryant & 3 & 7,89 \\
Questionário de Empatia Conjugal (QEC) & 2 & 5,26 \\
Escala de Empatia focada em grupo & 2 & 5,26 \\
Inventário de Empatia (IE) & 2 & 5,26 \\
IMHSC & 2 & 5,26 \\
Escala de Empatia Infanto-juvenil (EEmpa-IJ) & 1 & 2,63 \\
Questionário de coeficiente de Resiliência & 1 & 2,63 \\
Medida de inteligência emocional (MIE) & 1 & 2,63 \\
PROM- Pro-social & 1 & 2,63 \\
Análise de Performance e importância (API) & 1 & 2,63 \\
Escala de contágio Emocional & 1 & 2,63 \\
\hline
\end{tabular}

O Sistema de Avaliação de Habilidades Sociais (SSRS-BR) foi um dos instrumentos mais utilizados nos estudos de psicologia com participantes de pesquisa na área educacional com um total de 4 estudos (Cosenza, Pereira, \& Rabello, 2010; Del Prette, Barreto \& Freitas, 2011; Freitas \& Del Prette, 2010; Lopes, Del Prette, \& Del Prette, 2013). Ressalta-se que nos referidos estudos a empatia faz parte de uma sub-escala que está presente nos programas de pesquisa e intervenção.

Observa-se ainda que os estudos que utilizam o Índice de Empatía para Niños y Adolescentes (IECA) tratam da Escala de Empatia de Bryant (1982), na versão e validação de estudos para a população brasileira. Esta escala apresenta 22 itens que obtém uma medida de empatia experimentada por adolescentes em distintas situações. A Escala de Empatia de Bryant (1982) foi utilizada nos estudos de Rodrigues e Ribeiro (2011) e Ferreira, Del Prette e Lopes (2009).

Quanto ao Questionário de Empatia Conjugal (QEC), observa-se o artigo de Oliveira, Falcone e Ribas Jr. (2009) que realizaram uma avaliação das relações entre a empatia e a satisfação conjugal. Neste estudo, os autores utilizaram três medidas: O QEC, O IRI e a medida de satisfação conjugal. O outro artigo capturado, que também utilizou o QEC, trata-se da pesquisa de Sardinha, Falcone e Ferreira (2009) que apontam as relações entre a satisfação conjugal e as habilidades sociais percebidas no cônjuge. Ressalta-se que o artigo de Oliveira 
et al. (2009) realizou uma análise de regressão múltipla onde o resultado apontou forte relação entre a empatia do cônjuge e a satisfação conjugal.

Há ainda a Escala de Empatia Focada em Grupos utilizada nos estudos de Galvão, Camino, Gouveia e Formiga (2010) com bons critérios de validade, aplicada na realidade brasileira em estudantes do Ensino Médio. Segundo os autores, a empatia focada em grupos refere-se à capacidade afetiva das pessoas sentirem e pensarem o sofrimento de um determinado grupo específico.

Destaca-se ainda a importância do Inventário de Empatia (IE) de Falcone et al. (2008) que apresentaram, em seu estudo, uma Escala Brasileira de Empatia, do tipo Likert, baseada nos componentes cognitivos, afetivos e comportamentais da empatia em 715 universitários brasileiros. Esta escala também é utilizada em dois estudos capturados em nossa análise bibliográfica.

O Inventário Multimídia de Habilidades Sociais para Crianças (IMHSC) trata-se de um inventário de habilidades sociais onde um dos fatores de avaliação é a empatia e civilidade. Duas pesquisas utilizaram este inventário: (1) Del Prette et al. (2011), na área de psicologia e (2) Silva, Batista, Oliveira e Dassie-Leite (2012) na área de fonoaudiologia.

A Escala Initial psychometric analysis of an child and youth scale de KirstConceição e Martinelli (2014), com validação para a população brasileira com o nome de Escala de Empatia Infanto-juvenil (EEmpa-IJ), foi construída com base na literatura sobre empatia e de quatro escalas encontradas na literatura internacional e nacional. O estudo de Kirst-Conceição e Martinelli (2014) teve o objetivo de descrever o desenvolvimento e a validação inicial de uma escala de empatia para a população infanto-juvenil de estudantes brasileiros.

O questionário de coeficiente de Resiliência foi utilizado em uma pesquisa na área de saúde, sendo os participantes da pesquisa enfermeiros (Belancieri, Beluci, Silva, \& Gasparelo, 2010). Ressalta-se que embora trata-se de uma escala de resiliência, a empatia faz parte da organização do questionário com sendo um dos 7 fatores da resiliência.

A Medida de inteligência emocional (MIE) foi utilizada por Woyciekoski e Hutz (2010) em uma pesquisa em que examinou a associação entre as duas dimensões da personalidade (neuroticismo e extroversão) com a inteligência emocional. Ressalta-se que a medida de inteligência emocional apresenta 5 subescalas, sendo a empatia uma das subescalas.

D'Aurea-Tardeli (2008) realizou um estudo utilizando o Reasoning objective Messure (PROM - Pró-social). O PROM é uma medida objetiva que avalia o nível de raciocínio moral pró-social. O estudo objetivou relacionar à manifestação de solidariedade com, o sentimento de empatia, o juízo moral, o autoconceito e o projeto de vida.

A Análise de Performance e Importância (API) trata-se de um instrumento que tem por objetivo medir a qualidade de um serviço e satisfação do cliente, sendo o construto empatia um dos atributos da qualidade de um serviço. O API é 
um procedimento que mostra a importância relativa dos diversos atributos e o desempenho da empresa / prestador de serviços.

Finalmente, Gouveia, Guerra, Santos, Rivera e Singelis (2007) realizaram uma adaptação para o contexto brasileiro da Escala de Contágio Emocional. Neste artigo, os autores descrevem a diferença entre empatia e contágio emocional, sendo que a empatia envolve uma conscientização do outro e da sua experiência emocional, enquanto que o contágio emocional não exige esta consciência, mas apenas a vivência de uma emoção similar.

\section{Participantes da pesquisa}

$\mathrm{Na}$ Tabela 4, temos o quantitativo de estudos sobre empatia por participantes de pesquisa. A distribuição dos estudos ao longo do ciclo vital foi: adultos 28 estudos $(53,84 \%)$, crianças 11 estudos $(21,15 \%)$, adolescentes 7 estudos (13,46\%), jovens e adultos 4 estudos (7,69\%), idoso 1 estudo $(1,92 \%)$. Assim, constata-se um quantitativo bastante significativo nos estudos da empatia na fase adulta da vida, sendo que em idoso foi realizado somente 1 estudo o que evidencia um campo a ser estudado. Ressalta-se, ainda, o estudo de Silva (2016) que utilizou um protocolo de pesquisa par avaliar empatia e comportamento pró-social em ratos Wistar.

Tabela 4. Quantitativo de estudos sobre empatia por participantes de pesquisa

\begin{tabular}{ccr}
\hline Área & Quantitativo & $\%$ \\
\hline Adultos & 28 & 53.84 \\
Crianças & 11 & 21,15 \\
Adolescentes & 7 & 13,46 \\
Jovens e adultos & 4 & 7,69 \\
Idoso & 1 & 1.92 \\
Ratos & 1 & 1,92 \\
\hline
\end{tabular}

Nas pesquisas onde os participantes são adultos, em relação às áreas de atuação, como apontado anteriormente, há um grande interesse na área da saúde, com estudos em diversos campos de atuação, tais como em medicina, enfermagem, fisioterapia. Um aspecto importante a destacar é o fato de que há trabalhos onde o construto empatia é abordado tanto no aspecto da humanização na relação paciente-profissional quanto em trabalhos que avaliam o atendimento de hospitais, programas de saúde e satisfação com o serviço prestado pelos usuários do Sistema de Saúde.

Quanto à concentração de estudos em adultos, observa-se a seguinte distribuição: 09 pesquisas com usuários participantes de programas de atendimento $(17,30 \%) ; 08$ profissionais da saúde $(15,38 \%), 08$ pesquisas com estudantes universitários $(15,38 \%) ; 02$ pesquisas específicas de casais $(3,84 \%)$; 
01 pesquisa com médicos docentes de uma faculdade de medicina (1,92\%) e; 01 pesquisa com terapeutas e um estudante de psicologia $(1,92 \%)$.

Nas pesquisas que tratam de satisfação de usuários de um programa de atendimento temos: $2(3,84 \%)$ participantes usuários de um hospital; 2 (3,84\%) que faz um estudo com usuários e funcionários de um programa de saúde em odontologia; $2(3,84 \% \%)$ que trata do Programa de saúde da família; $1(1,92 \%)$ participantes usuários de fisioterapia; 1(1,92\%) participantes de cirurgia cardíaca; $1(3,84 \%)$ trata de usuários de um programa de exercício físico e saúde. Ressalta-se que o instrumento utilizado nas pesquisas sobre a satisfação dos usuários nos serviços prestados foi a Escala Servqual.

Quanto aos 08 estudos (15,38\%) com participantes adultos que são profissionais da saúde temos: 04 estudos (7,69\%) que avaliam o grau de empatia em enfermeiros e técnicos de enfermagem; 01 estudo (1,92\%) sobre a relação terapeuta e paciente; 01 estudo $(1,92 \%)$ com médicos e professores de cursos de medicina; 01 estudo $(1,92 \%)$ sobre relação humanizada de fisioterapeuta e seus pacientes; 1 estudo (1,92\%) que trata de empatia e coeficiente de resiliência em enfermeiros.

Quanto aos 08 artigos (15,38\%) capturados nesta análise com os participantes universitários, observou-se o quantitativo de 07 estudos $(13,46 \%)$ realizados para validar e adaptar escalas de empatia. As escalas utilizadas foram: a Escala Multidimensional de Reatividade Interpessoal, o Inventário de Empatia (IE), (EMRI) e a Escala de Contágio Emocional. Outra escala que foi utilizada nesta população foi a Escala SERVQUAL com o objetivo de avaliar a qualidade de serviços prestados aos alunos universitários. Ressalta-se 01 estudo (1,92\%) de Aguiar, Formiga e Cantinilo (2017) que investigou a associação entre empatia e personalidade na população de estudantes de medicina.

Costa e Azevedo (2010) realizaram uma pesquisa qualitativa, utilizando entrevista semiestruturada com participantes médicos docentes de uma faculdade de medicina. Os autores consideram que a empatia pode enriquecer a prática médica e cogitam a possibilidade de ensinar a ser empático na Relação Médico-paciente (RPM).

As 02 pesquisas com casais $(3,84 \%)$ tratam da relação da empatia e satisfação conjugal. Em uma das pesquisas Oliveira, Falcone e Ribas Jr (2009) utilizam o QEC, o IRI e medida de satisfação conjugal e realizam uma análise de regressão múltipla apontando forte ligação entre a empatia e satisfação conjugal. Os autores apontam que devido ao coeficiente alfa elevado $(0,95)$ encontrado na medida do QEC permite recomendar a utilização dessa escala na prática clínica e em pesquisa. O estudo de Sardinha, Falcone e Ferreira (2009) também apontam forte relação entre a empatia do cônjuge e a satisfação conjugal, seguida pela expressão de sentimentos e defesa dos próprios direitos. Ressalta-se que nessa pesquisa os dados apresentados identificam que o número de filhos se correlacionou inversamente com a satisfação e as mulheres foram percebidas 
como mais empáticas. Assim, Sardinha et al (2009) concluem que as habilidades sociais, especialmente a empatia, parecem ser facilitadores da satisfação conjugal e recomendam o desenvolvimento dessas habilidades no tratamento de casais em crise.

Nos 11 estudos $(21,15 \%)$ com crianças a idade dos participantes da pesquisa variaram desde bebês com menos de 18 meses até a idade de 12 anos. As escalas mais utilizadas nesta população foram: Escala de Empatia de Bryant, IMHSC, SSRS. Ressalta-se um estudo $(1,92 \%)$ realizada na área da saúde, em fonoaudiologia, com o objetivo de avaliar as habilidades sociais de crianças disfônicas (Silva, et al., 2012). Todos os outros 09 estudos (17,30\%) foram da área de psicologia.

Os 07 estudos $(13,46 \%)$ com participantes adolescentes realizam validação e estudos de consistência interna de escalas. As escalas utilizadas nestas pesquisas foram: IRI, Escala de Empatia Focada em Grupo, PROM- Prosocial.

Quanto aos 04 artigos $(7,69 \%)$ que utilizam em sua pesquisa participantes jovens e adultos, observou-se uma grande tendência em estudos para adaptar e validar as escalas empregadas para o contexto brasileiro e para esta população. As escalas mais utilizadas foram: IRI, Escala de Empatia Focada em Grupo e PROM- Pro-social.

Ao longo do ciclo vital observa-se, na revisão de nossos estudos sobre o construto empatia, que somente 1 artigo $(1,92 \%)$ realizou sua pesquisa com participante idoso (Ramos, Pedrão, \& Furegato, 2009). O objetivo do estudo foi analisar a interação de um profissional com um cuidador de uma pessoa idosa, com base na teoria da relação de ajuda não-diretiva.

\section{DISCUSSÃo}

O presente estudo teve o objetivo de analisar as publicações e os resultados encontrados à luz da literatura do construto empatia no período de 2007 a 2017. Foram analisadas as seguintes categorias: quantitativo de publicações por ano, áreas de publicações, os instrumentos mais utilizados nas pesquisas e quais foram os participantes de pesquisa.

Quanto ao quantitativo de artigos publicados, destaca-se uma concentração maior de estudos empíricos publicados no período de 2007 a 2012, sendo o ano de 2010 aquele que apresentou maior quantitativo de estudos. Observa-se, em 2010, uma preocupação dos psicólogos brasileiros em validar as traduções das escalas para a população brasileira, sendo realizadas, na grande maioria dos estudos, validações de instrumento e medidas de empatia para nossa população em diferentes grupos. Ressalta-se que, no período de 2012 a 2017, houve uma queda nos estudos empíricos sobre empatia o que nos leva a refletir que diante das diversas validações de escalas, nos anos anteriores, 
ocorreu uma queda de interesse dos pesquisadores em estudar e validar escalas para medir esse construto.

Quanto à área de publicação, o maior quantitativo de estudos refere-se a área de psicologia, com destaque para os estudos de análise do construto e validações de estudos. A segunda maior área foi da saúde, que apresentou estudos com objetivo de investigar a relação empática estabelecida nos atendimentos realizados pelos profissionais da área da saúde com os usuários. Observou-se ainda a preocupação, na área da saúde, com a qualidade de serviços prestados de diversos programas de atendimento aos usuários e a preocupação com atendimentos mais humanizados onde a empatia é um construto investigado.

Ressalta-se que na área educacional somente dois artigos foram publicados. Os referidos artigos apresentavam o objetivos de avaliar a qualidade de serviços prestados aos usuários de instituições de ensino, sendo a relação empática no atendimento um construto determinante na qualidade de serviço, ou seja, não encontramos trabalhos com o objetivo de medir as dimensões da empatia nos discentes ou docentes de instituições de ensino. É importante ressaltar que não detectamos estudos que correlacionassem a relação da empatia no processo ensino-aprendizagem.

Quanto aos instrumentos de medidas, o presente estudo mostra que a visão da empatia, como constructo multidimensional, é a mais adotada entre os psicólogos brasileiros. O instrumento mais utilizado nas pesquisas foi o a Escala Multidimensional de Reatividade Interpessoal (EMRI), escala validada e traduzida no Brasil da Interpersonal Reactivity Index (IRI) de Davis (1980), bem como as traduções deste instrumento com validação desta escala no contexto nacional. Assim, a escolha por pesquisadores brasileiros desta escala mostra a sua relevância teórica e sua consistência empírica. Ressalta-se que este resultado encontra-se de acordo com a literatura internacional, pois o IRI de Davis (1980) é a escala mais utilizada para medir empatia encontrada na literatura internacional.

Em relação a área de Saúde, os dados apontam a importância que tem sido atribuída à empatia, nesse contexto, com objetivo de medir a qualidade de serviços prestados aos usuários. Assim, o instrumento mais utilizado para medir a qualidade de prestação de serviço foi o SEVQUAL, sendo a empatia um dos fatores determinantes para a qualidade de serviço. Ressalta-se que a humanização no atendimento é uma das preocupações na formação e na prática profissional e o interesse pelo estudo da empatia converge para essa preocupação. A empatia é estudada no ensino universitário, nos diversos setores de atendimento, na relação médico - paciente, no Programa de Saúde da Família e nos atendimentos hospitalares.

Quanto aos participantes de pesquisa, observa-se um quantitativo significativo de estudos realizados em adultos. Na grande maioria os estudos 
com esta população relacionam-se aos atendimentos na área da saúde com o objetivo de avaliar a qualidade de serviço de um programa de atendimento, bem como avaliar o atendimento humanizado. Observou-se, ainda, estudos que analisam a relação empáticas em casais.

Quanto aos artigos com participantes adolescentes, ressalta-se 0 quantitativo significativo de estudos que realizam validações de escalas para a população brasileira. Observa-se que há uma tendência, nestas pesquisas, em serem aplicadas em estudantes do ensino médio e em universitários, possibilitando um quantitativo alto de participantes de ambos os sexos. Ressaltase, que para validar um instrumento, é importante um grande quantitativo de participante o que nos leva a entender a tendência dos pesquisadores em aplicar a escala em escolas e universidades.

Nos artigos que os participantes são crianças a idade dos participantes de pesquisa são desde bebê com menos de 8 meses até crianças com idade de 12 anos. Somente um artigo foi com participantes bebês com o objetivo de verificar a relação empática do bebê e o cuidador através de observação sistemática. Os estudos com crianças aplicaram a Escala de empatia de Bryant, com objetivo de avaliar as habilidades sociais de crianças. Outra escala aplicada nos estudos com crianças foi a SSRS-BR, que avalia as habilidades sociais em crianças sendo a empatia uma subescala.

Assim, podemos observar que os dados desta análise do perfil da produção científica e medidas mais utilizadas em pesquisa apontam para necessidade de um número maior de estudos no contexto educacional. Diante da importância da empatia para o desenvolvimento dos indivíduos, em seus vários aspectos, é importante sinalizar que tão pouco tenha sido feito em relação a influência da empatia nas questões educacionais. Ressalta-se que foi encontrado somente um trabalho relacionado à empatia do professor em relação aos alunos, sendo esta pesquisa realizada na área de educação em saúde.

É importante refletir sobre as razões de não haver um questionamento maior a respeito da relação de empatia entre professores e alunos. Assim, a relação empática entre professores e alunos nos indica como uma área ainda a ser explorada por aqueles que têm interesse no estudo da empatia. A relação empática afetaria o desempenho dos alunos? Teria efeitos como diminuir ou reduzir o estresse de professores e o burnout? São algumas das questões ainda a serem abordadas nos estudos sobre empatia na área educacional.

De um modo geral, essa revisão mostra que esse é um campo que pode e deve ser explorado por pesquisadores nacionais em pesquisas futuras. Tendo em vista os poucos estudos com amostras brasileiras em diferentes etapas do ciclo da vida e em diferentes contextos. Ressalta-se ainda a importância de realizar estudos com intervenções que visem o desenvolvimento do construto empatia, sendo estas intervenções um campo de estudo que foi pouco investigado na população brasileira. 


\section{CONSIDERAÇÕES FINAIS}

A análise bibliográfica do construto empatia ofereceu informações importantes sobre esse campo de estudo. Verificou-se, na presente revisão, que os trabalhos encontrados apresentam foco maior em estudos na área da psicologia, em seus mais diversos campos de estudo, tais como: psicologia social, psicologia do desenvolvimento, bem como estudos de validação de escalas. A área da saúde também apresenta estudos voltados para este construto, sendo que o objetivo maior nesta área é verificar a qualidade de serviços prestados aos usuários da saúde bem como a humanização dos atendimentos.

É importante refletir sobre as razões de não haver estudos na área educacional que aprofundem temas relacionados ao construto empatia, principalmente no que se refere à relação de empatia entre professores - alunos e sua relação com o processo ensino-aprendizagem. Assim, a relação empática entre professores e alunos no ambiente educacional nos indica uma área ainda a ser explorada por aqueles que têm interesse na área educacional e nos estudo da empatia.

\section{DECLARAÇÃO DE CONFLITOS DE INTERESSE}

Não há conflitos de interesse.

\section{REFERÊNCIAS}

Aguiar, C. S., Formiga, N. S., \& Cantinilo, A. (2017). Personality traits and empathic abilities: A predictive study on medical students. Boletim: Academia Paulista de Psicologia, 37(92), 129-138.

Batson, C. D., Fultz, J., \& Schoenrade, P. A. (1987). Distress and empathy: Two qualitatively distinct vicarious emotions with different motivational consequences. Journal of Personality, 55, 19-39. doi:10.1111/j.14676494.1987.tb00426.x.

Belancieri, M. F., Beluci, M. L., Silva, D. V. R., \& Gasparelo, E. A. (2010). A resiliência em trabalhadores da área da enfermagem. Estudos de Psicologia (Campinas), 27(2), 227-233. doi:10.1590/S0103-166X2010000200010.

Bryant, B. K. (1982). An index of empathy for children and adolescents. Child Development, 53(2), 413-425. doi:10.2307/1128984

Castro, M. S., Silva, N. L., Monteiro, W., Palma, A., \& Resende, H. G.. (2010). Motivos de permanência dos praticantes nos programas de exercícios físicos oferecidos pelo Serviço Social do Comércio - Brasil. Motricidade, 6(4), 2333.

Recuperado

de

http://www.scielo.mec.pt/scielo.php?script=sci arttext\&pid=S1646$107 \times 2010000400004 \&$ lng $=$ pt\&tlng=pt. 
Cosenza, R. M., Pereira, D. J., \& Rabello L. F. M. F. (2010). Resolução de problemas interpessoais: Promovendo o desenvolvimento sociocognitivo na escola. Psicologia em Estudo, 15(4), 831-839. http://dx.doi.org/10.1590/S1413-73722010000400019.

Costa, F. D., \& Azevedo, R. C. S. (2010). Empatia, relação médico-paciente e treinamento médico: Uma visão qualitativa. Revista Brasileira de Educação Médica, 34(2), 261-269. doi:10.1590/S0100-55022010000200010.

Cox, C. L., Uddin, L. Q., Di Martino, A., Castellanos, F. X., Milham, M. P., \& Kelly, C. (2012). The balance between feeling and knowing: Affective and cognitive empathy are reflected in the brain's intrinsic functional dynamics. Social Cognitive and Affective Neuroscience, 7(6), 727-737. doi: $10.1093 /$ scan/nsr051.

D'Aurea-Tardeli, D. (2008). A manifestação da solidariedade em adolescentes: Um estudo sobre a personalidade moral. Psicologia: Ciência e Profissão, 28(2), 288-303. doi:10.1590/S1414-98932008000200006.

Damásio, A. R. (2009). Em busca de Espinosa: Prazer e dor na ciência dos sentimentos. São Paulo, SP: Companhia das Letras.

Davis, M. H. (1980). A Multidimensional approach to individual differences in empathy. Catalog of Selected Documents in Psychology, 10, p. 85. Recuperado

de http://www.ucp.pt/site/resources/documents/ICS/GNC/ArtigosGNC/Alexand reCastroCaldas/24 Da80.pdf.

Davis, M. H. (1983a). Measuring individual difference in empathy: Evidence for a multidimensional approach. Journal of Personality and Social Psychology, 44, 113-126. doi:10.1037/0022-3514.44.1.113

Davis, M. H. (1983b). The effects of dispositional empathy: Evidence for a multidimensional approach. Journal of Personality and Social Psychology, 51(2), 167-184. doi:10.1111/j.1467-6494.1983.tb00860.x

Davis, M. H., \& Oathout, H. A. (1992). The effect of dispositional empathy on romantic relationship behaviors: Heterosocial anxiety as a moderating influence. Journal of Personality and Social Psychology Bulletin, 18, 76-83. doi: $10.1177 / 0146167292181011$

De Greck M., Wang G., Yang X., Wang X., Northoff G., \& Han, S. (2012). Neural substrates underlying intentional empathy. Social Cognitive and Affective Neuroscience, 7(2), 135-144. doi:10.1093/scan/nsq093

Del Prette, Z. A. P., Barreto, S., \& Freitas, L. (2011). Habilidades sociais na comorbidade entre dificuldades de aprendizagem e problemas de comportamento: Uma avaliação multimodal. Psico, 42(4), 503-510. Recuperado de http://revistaseletronicas.pucrs.br/ojs/index.php/revistapsico/article/view/7 593/7457. 
Falcone, E. (1999). A avaliação de um programa de treinamento da empatia com universitários. Revista Brasileira de Terapia Comportamental e Cognitiva, 1(1), 23-32. Recuperado de http://pepsic.bvsalud.org/scielo.php?script=sci arttext\&pid=S1517$55451999000100003 \& \operatorname{lng}=p t \& t \operatorname{lng}=p t$.

Falcone, E. M. O., Gil, D. B., \& Ferreira, M. C. (2007). Um estudo comparativo da frequência de verbalização empática entre psicoterapeutas de diferentes abordagens teóricas. Estudos de Psicologia (Campinas), 24(4), 451461. doi: 10.1590/S0103-166X2007000400005.

Falcone, E. M. O., Ferreira, M. C., Luz, R. C. M., Fernández, C. S, Faria, C. A., \& D'Augustin, J. F. (2008). Inventário de Empatia (IE): Desenvolvimento e validação de uma medida brasileira. Avaliação Psicológica, 7(3), 321-334. Recuperado de http://pepsic.bvsalud.org/pdf/avp/v7n3/v7n3a06.pdf.

Falcone, E. M. O., Pinho, V. D. de, Ferreira, M. C., Fernandes, C. S., D'Augustin, J. F., Krieger, S. ... Pinheiro, L. C. (2013). Validade convergente do Inventário de Empatia (IE). Psico-USF, 18(2), 203-209. doi: $10.1590 / S 1413-82712013000200004$.

Ferreira, B. C., Del Prette, Z. A. P., \& Lopes, D. C. (2009). Habilidades empáticas de crianças videntes e cegas e a possível influência de variáveis sociodemográficas. Interação em Psicologia, 13(1), p. 49-58. Recuperado de https://revistas.ufpr.br/psicologia/article/download/11745/10485.

Formiga, N. S. (2012, 29 de setembro). Os estudos sobre empatia: Reflexões sobre um construto psicológico em diversas áreas científicas. Portal Psicologia.pt.

Recuperado

de http://www.psicologia.pt/artigos/textos/A0639.pdf.

Formiga, N. S., Rique, J., Galvão, I., Camino, C., Mathias, A., \& Medeiros, F. (2011). Escala Multidimensional de Reatividade Interpessoal - EMRI: consistência estrutural da versão reduzida. Revista Psicologia Trujill, 13(2), 171-184.

Recuperado de http://revistas.ucv.edu.pe/index.php/R PSI/article/download/171/83.

Freitas, L. C., \& Del Prette, Z. A. P. (2010). Validade de critério do sistema de avaliação de habilidades sociais (SSRS-BR). Psicologia: Reflexão e Crítica, 23(3), 430-439. doi:/10.1590/S0102-79722010000300003.

Freud, S. (2006). Os chistes e sua relação com o inconsciente. In J. Salomão (Trad.), Edição Standard das obras psicológicas completas de Sigmund Freud. (vol. 8). Rio de Janeiro, RJ: Imago. (Trabalho original publicado em 1905).

Galvão, L. K. S., Camino, C. P. S., Gouveia, V. V., \& Formiga, N. S. (2010). Proposta de uma medida de empatia focada em grupos: Validade fatorial e consistência interna. Psico-PUCRS, 41(3), 399-405. Recuperado de http://revistaseletronicas.pucrs.br/ojs/index.php/revistapsico/article/view/5 923/5862. 
Goleman, D. (1995). Inteligência emocional. Rio de Janeiro, RJ: Objetiva

Gouveia, V. V., Guerra, V. M., Santos. W. S., Rivera, G. A., \& Singelis, T. M. (2007). Escala de Contágio Emocional: adaptação ao contexto Brasileiro. Psico-PUCRS, 38(1), 45-54. Recuperado de http://revistaseletronicas.pucrs.br/ojs/index.php/revistapsico/article/view/1 923/1429.

Harzing, A. W. (2007). Google Scholar: A new data source for citation analysis. Ethics in Science and Environmental Politics, 8(1), 61-73. doi: $10.3354 /$ esep00076.

Hoffman, M. (1981). Is altruism part of human nature? Journal of Personality and Social Psychology, 40, 121-137. doi:10.1037/0022-3514.40.1.121

Hoffman, M. (1990). The contribution of empaty to justice and moral judgment. In N. Eisenberg, \& J. Strayer (Eds.), Empathy and its development (pp. 4780). Cambridge, MA: Cambridge University Press.

Hora, H. R. M., Moura, L. A. T., \& Vieira, G. B. S. V. (2009). Análise da qualidade de serviços de um shopping center, na percepção dos clientes internos. Revista Eletrônica Produção \& Engenharia, 2(2), 126-138. Recuperado de http://www.fmepro.org/ojs/index.php/rpe/article/view/141/72.

Ickes, W. (1997). Introduction. In W. Ickes (Ed.), Emphatic accuracy (pp. 1-16). New York, NY: The Guilford Press.

Ickes, W. \& Simpson, J. A. (1997). Managing empathic accuracy in close relashionships. In W. Ickes (Ed.), Emphatic accuracy (pp. 218-250). New York, NY: The Guilford Press.

Kirst-Conceição, A. da C., \& Martinelli, S. de C. (2014). Análises psicométricas iniciais de uma escala de empatia infantojuvenil (EEmpa-IJ). Avaliação Psicológica, 13(3), 351-358. Recuperado de http://pepsic.bvsalud.org/pdf/avp/v13n3/v13n3a07.pdf.

Koller, S. H., Camino, C., \& Ribeiro, J. (2001). Adaptação e validação de duas escalas de empatia para uso no Brasil. Estudos de Psicologia, 18(3), 43-53. http://dx.doi.org/10.1590/S0103-166X2001000300004.

Lopes, D. C.; Del Prette, Z. A. P., \& Del Prette, A. (2013). Recursos multimídia no ensino de habilidades sociais a crianças de baixo rendimento acadêmico. Psicologia Reflexão e Crítica, 26(3), 451-458. doi:10.1590/S010279722013000300004.

Oliveira, M., Falcone, E., \& Ribas Jr., R. (2009). A avaliação das relações entre a empatia e a satisfação conjugal: Um estudo preliminar. Interação em Psicologia, 13(2). doi:10.5380/psi.v13i2.8025.

Oliveira, O. J., \& Ferreira, E. C. (2008). Adaptação e aplicação da escala SERVQUAL na educação superior. Gestão da Produção, Operações e Sistemas - GEPROS, 3(3), 133-146. https://doi.org/10.15675/gepros.v3i3.473. 
Parasuraman, A., Zeithami, V. A., \& Berry, L. L. (1988). ServQual: A multipleitem scale for measuring consumer peceptions of service quality. Journal Retailing, 64(1), 12-40.

Prass, R. M., Sant'Anna, L. C., \& Godoy, L. P. (2010). Avaliação da qualidade de serviços prestados na área educacional através do modelo Servqual. Revista Gestão Industrial, 6(2), 213-231. doi:10.3895/S1808-04482010000200012 Ramos, T., M., B., Pedrão, L. J., \& Furegato, A., R., F. (2009). A relação de ajuda não-diretiva junto ao cuidador de um idoso incapacitado. Revista Eletrônica de Enfermagem, 11(4), 923-31. Recuperado de https://www.fen.ufg.br/revista/v11/n4/pdf/v11n4a18.pdf.

Rodrigues, M. C., \& Ribeiro, N. N. (2011). Avaliação da empatia em crianças participantes e não participantes de um programa de desenvolvimento sociocognitivo. Psicologia: Teoria e Prática, 13(2), 114-126. Recuperado de http://pepsic.bvsalud.org/pdf/ptp/v13n2/v13n2a09.pdf.

Rogers, C. R. (1992). Terapia centrada no cliente. São Paulo, SP: Martins Fontes. Rogers, C. R. (2001). Tornar-se pessoa (5.ed.). São Paulo, SP: Martins Fontes.

Sampaio, L. R., Camino, C. P. dos S., \& Roazzi, A. (2009). Revisão de aspectos conceituais, teóricos e metodológicos da empatia. Psicologia: Ciência e Profissão, 29(2), 212-227. doi:10.1590/S1414-98932009000200002.

Sampaio, L. R., Guimarães, P. R. B, Camino, C. P. S., Formiga, N. S., \& Menezes, I. G. (2011). Estudos sobre a dimensionalidade da empatia: Tradução e adaptação do Interpersonal Reactivity Index (IRI). Psico, 42(1), 67-76. Recuperado http://revistaseletronicas.pucrs.br/ojs/index.php/revistapsico/article/view/6 456/6302.

Sardinha, A., Falcone, E. M. de O., \& Ferreira, M. C. (2009). As relações entre a satisfação conjugal e as habilidades sociais percebidas no cônjuge. Psicologia: Teoria e Pesquisa, 25(3), 395-402. doi:10.1590/S010237722009000300013.

Silva, P. R. R. da. (2016). Empatia e reciprocidade em ratos Wistar: Um paradigma para avaliar comportamento pró-social. (Dissertação em Mestrado). Universidade Federal do Rio Grande do Norte, Natal. Recuperado de https://repositorio.ufrn.br/jspui/handle/123456789/21584?locale=en.

Silva, M., Batista, A. P., Oliveira, J. P., \& Dassie-Leite, A. P. (2012). Habilidades sociais em crianças disfônicas. Jornal da Sociedade Brasileira de Fonoaudiologia, 24(4), 361-367. doi:10.1590/S2179-64912012000400012.

Woyciekoski, C., \& Hutz, C. S. (2010). Inteligência emocional avaliada por autorrelato difere do construto personalidade? Psico-USF, 15(2), 151-159. doi: 10.1590/S1413-82712010000200003. 
Sobre os autores

Sonia Maria Lourenço de Azevedo é Psicóloga pela Universidade Gama Filho, Mestre e Doutoranda em Psicologia pela Universidade Salgado de Oliveira UNIVERSO. Linha de pesquisa em Psicologia Social e recebeu financiamento de pesquisa da CAPES. E-mail, sonietka@uol.com.br Márcia Maria Peruzzi Elia da Mota é Psicóloga, Doutora em Psicologia pela Universidade de Oxford- Inglaterra, Professora do Programa de Pós-Graduação em Psicologia da Universidade Salgado de Oliveira - UNIVERSO e Professora do Programa de Pós-Graduação em Psicologia Social da Universidade do Estado do Rio de Janeiro-UERJ, Bolsista de Produtividade nível 2 do CNPq. E-mail, mmotapsi@gmail.com.br

Marsyl Bulkool Mettrau é Doutora em Psicologia da Educação pela Universidade do Minho- Portugal, PhD em Educação pela Universidade Federal Fluminense UFF. Professora do Programa de Pós- Graduação em Psicologia da Universidade Salgado de Oliveira - UNIVERSO. E-mail, marsylmettrau@gmail.com

Certificamos que todos os autores participaram suficientemente do trabalho para tornar pública sua responsabilidade pelo conteúdo. A contribuição de cada autor pode ser atribuída como se segue: Sonia Maria Lourenço de Azevedo (autor A), Márcia Mota (autor B) e Marsyl Bulkool Mettrau (autor C) contribuíram para a conceitualização e delineamento do trabalho apresentado. Sonia Maria Lourenço de Azevedo (autor A) realizou ainda o levantamento e análise dos dados. Sonia Maria Lourenço de Azevedo (autor A) e Márcia Mota (autor B) fizeram a redação do artigo inicial e final.

O financiamento foi da bolsa de doutorado da CAPES atribuída a primeira autora (Sonia Maria Lourenço de Azevedo).

Recebido em: 01/06/2016

Revisado em: 30/01/2017

Aceito em: 24/02/2018 